\title{
Radio burst from converging separatrices
}

\author{
H. Aurass, G. Rausche, and G. Mann
}

\author{
Astrophysikalisches Institut Potsdam (AIP), An der Sternwarte 16, 14482 Potsdam, Germany \\ e-mail: haurass@aip.de
}

Received 26 March 2007 / Accepted 26 June 2007

\begin{abstract}
Context. In a previous paper on the X17 flare October 28, 2003, we found, among other radio flare sources, one displaced at $\approx 0.2 R_{\odot}$ north of the flaring AR 10486, away from the main $\mathrm{H} \alpha$ and hard X-ray flare emission. The source came to our attention due to its timing, its spatial displacement from the flaring active region, and due to the behavior of the radio spectral fine structure sources which are embedded in the continuum emission. We speculated that the source is situated at a separator of coronal magnetic flux systems. Aims. Here we analyze the topology of the potential coronal magnetic field extrapolated from SOHO-MDI data near the source site of this meter-decimeter radio continuum.

Methods. Using the Source Method for magnetic field modeling, and the displacement gradient of the field lines as a connectivity measure, we give a description of the critical field structure. We compare it with the Nançay Radio Heliograph source positions of the continuum which have been classified by the spectral data of Astrophysical Institute Potsdam.

Results. We find that the radio source occurs near the contact of three separatrix surfaces between magnetic flux cells. There are other separatrix surfaces in the field which are not distinguished by a strong radio source during the analyzed flare.

Conclusions. This is the first evidence for the occurence of a strong coronal radio burst continuum source at such coronal magnetic field structures.
\end{abstract}

Key words. Sun: corona - Sun: radio radiation - Sun: magnetic fields - Sun: flares - Sun: coronal mass ejections (CMEs) acceleration of particles

\section{Introduction}

The X17 flare and ground level event of October 28, 2003 in AR 10486 was analyzed in several respects (e.g. Aurass et al. 2006 (P1); Hurford et al. 2006). In P1 we demonstrated that the magnetic field structure in AR 10486, and also between this and the neighboring five other active regions is essential to understand the positions of the radio flare sources. Figure 1a presents a map of the SOHO-MDI 11:11 UT longitudinal component of the photospheric magnetic field, and the arrangement of active regions in this part of the solar disk.

The coronal magnetic field cannot be routinely measured. The potential or force-free extrapolation of the photospheric measurements gives a reasonable approach to its large-scale structure (e.g. Somov 2000, p. 414). It is evident (Spicer 1977) that in coronal heights which are not too large, the flare energy is released and streams of energetic particles are formed which later interact with the denser atmosphere (visible in e.g. $\mathrm{H} \alpha$ and hard X-rays), or escape into space. The accelerated particles propagate along field lines of the coronal magnetic field, and under certain circumstances flare burst radio emission is excited. Decimeter and meter wave sources of plasma emission are a diagnostic tool for the presence of $\approx 10 \mathrm{keV}$ electrons (Melrose 1985) in the lower corona - forming drift bursts and continuum emission in dynamic radio spectra as e.g. recorded by the AIP 40-800 MHz spectrometer (Mann et al. 1992). Radio source positions shown in this paper are derived from Nançay Radio Heliograph data (NRH, Kerdraon \& Delouis 1997). The halfpower width of the radio source of interest is $\approx 190$ arcsec. This is much larger than subtle structures of the magnetic field. Therefore in source site plots we only show the centroids of a
Gaussian fit of the source shape. In the present case the radio sources are situated on the disc, therefore the source height is unknown. In a previously published analysis of fiber burst fine structures in the spectral data of the same event (see P1) we found a five-fold Newkirk (1961) density model as appropriate in the lower corona for the time and spatial range of interest. This density model is also applied, henceforth, giving a height range between 45 and $95 \mathrm{Mm}( \pm 5 \mathrm{Mm})$ above the photosphere for the relevant radio sources.

In $\mathrm{P} 1$, we described two remote radio sources different from those near the $\mathrm{H} \alpha$ flare ribbons and the hard X-ray sources in AR 10486. One was shifted toward SW, dominating in the impulsive flare phase and during the acceleration of highly relativistic electrons. There are strong arguments that this source is due to the reconnection outflow termination shock (Aurass \& Mann 2004; Mann et al. 2006) and that it is connected with flarerelated, dynamically evolving magnetoplasma structures above and to the SW of AR 10486.

The second source will be considered here. It occurs near AR 10491 north of the flaring AR 10486 (Fig. 1b). Its flux profile is compared with the GOES flux curve ${ }^{1}$ in Fig. 2a. It is a decimetric-metric continuum (DMCS, henceforth) and occurs without associated effects in $\mathrm{H} \alpha$, EUV, and hard X-rays (given groundbased $\mathrm{H} \alpha-$, RHESSI HXR-, and TRACE EUV data). As shown in Fig. 2a the DMCS acts briefly early in the impulsive phase, and is strongest well thereafter. The source dominates the $\mathrm{dm}$ radio flux of the event during the start of the injection of high

\footnotetext{
${ }^{1}$ Off-scale from 11:06-11:15 UT. Figure 1 in Hurford et al. (2006) confirms a declining hard X-ray profile from RHESSI and INTEGRAL data past 11:09 UT.
} 


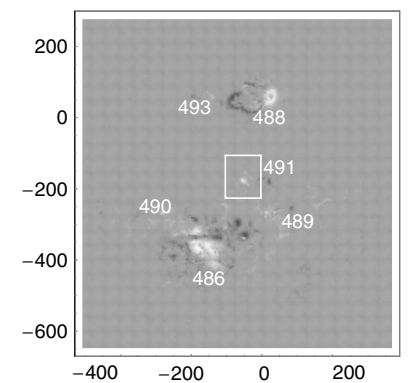

a)

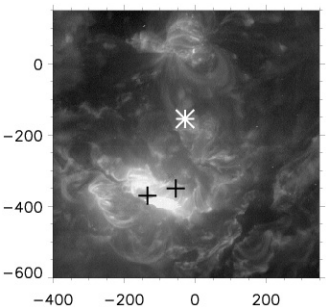

b)

Fig. 1. a) SOHO-MDI 11:11 UT magnetogram, $\mathrm{N}$ is top, $\mathrm{E}$ is left, axes in arcsec. Numbers: NOAA active regions as 10xxx. The white box encloses the field considered in Fig. 3b. b) SOHO-EIT $195 \AA$ image (11:47 UT). Asterisk: decimeter-meter continuum (DMCS). "+": RHESSI HXR footpoint source centroids in the flaring AR 10486 on 11:06 UT.

energy protons at the Sun (see the bar in Fig. 2a, and Klassen et al. 2005). Embedded in the DMCS spectrum are radio spectral fine structures - pulsations and patches of fiber bursts. In P1 we used the fiber bursts to determine those magnetic field lines of the potential field extrapolation of the SOHO MDI data (Fig. 1a) which are most compatible with the radio spectral fine structure parameters. Two such field lines are shown in a perspective view in Fig. 2b magnetically linking the northern part of AR 10486 with a footpoint in the trailing part of AR 10491 and from this place further to ARs 10488/10493. In the same figure we have inserted a white column representing the DMCS centroid for a height range corresponding to plasma frequencies between 432 and $327 \mathrm{MHz}$ (the both relevant NRH imaging frequencies).

As evidenced in P1, we can use the potential field extrapolation of the SOHO-MDI 11:11 UT data to describe the static large-scale magnetic field structures in the corona. Non-potential components occur either as smoothly-distributed or as highly concentrated electric currents (Somov et al. 2003). The concentrated currents flow in current layers between flux cell boundaries (at separatrices) or in reconnecting current layers where separatrices penetrate each other (on separator field lines). It is possible that the DMCS is a source which points on such a concentrated current flow in a complex structured potential field. The current occurs as a consequence of a disturbance of the field structure due to the flare in AR 10486. It yields a possible acceleration mechanism for electrons necessary for the DMCS formation. We now consider the magnetic field structure near the DMCS in detail.

\section{Analysis of the potential magnetic field near the radio source site}

Much work was devoted to the problem of magnetic connectivity in the corona (e.g. Gorbachev \& Somov 1988; Démoulin et al. 1996, 1997; Titov et al. 2002). Démoulin et al. (1997) compare the coronal field topology studied by observation-adapted magnetic monopole arrangements (the so-called Source Method) with the positions of $\mathrm{H} \alpha$ flare ribbons. Here, we apply the norm of the displacement gradient $N(x, y)$ in the photosphere as defined by Démoulin et al. (1997). The displacement gradient measures the change of the norm of vectors joining the start- and the endpoint of field lines for a small change of the starting point. Large values of $N$ mean that at the given field line starting range, neighboring start points have a large end-point distance. Small $N$ values mean that neighboring field line start points remain

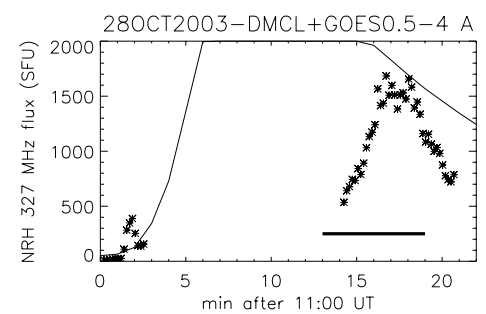

a)

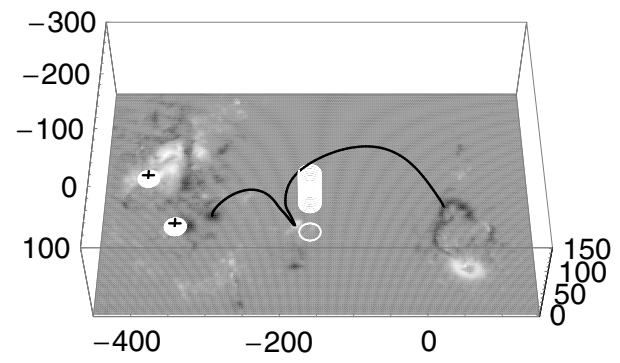

b)

Fig. 2. a) $327 \mathrm{MHz}$ flux of DMCS (*, in solar flux units $10^{-22} \mathrm{~W} \mathrm{~s} \mathrm{~m}^{-2}$ ) together with the normalized GOES 0.5-4 $\AA$ X-ray flux (off-scale from 11:06-11:15 UT). Note the flash at the impulsive onset, and the $6 \mathrm{~min}$ pulse in the main phase during proton injection onset (the dark bar, and Klassen et al. 2005). b) View from W, top is E, right is $\mathrm{N}$, on the magnetogram Fig. 1a with RHESSI HXR sources ("+" on white spot) and 2 field lines from fiber burst analysis (dark, see P1). White circle and column: projected site and height of the DMCS, see Fig. 1b.

neighbors at the end of the field lines. With $(x, y)$ as Cartesian coordinates in the photosphere, $\mathbf{X}$ denotes a field line start-endconnecting vector with the components $\left(X_{1}, X_{2}\right)$. Then $N(x, y)$ is

$N(x, y)=\sqrt{\sum_{i=1,2}\left(\left(\frac{\partial X_{i}}{\partial x}\right)^{2}+\left(\frac{\partial X_{i}}{\partial y}\right)^{2}\right)}$

Firstly, we computed $N(x, y)$ using the field line set obtained by potential extrapolation of the MDI magnetogram (courtesy: A. Hofmann, and see P1). The magnetogram has limited resolution, and some noise level. Therefore, we follow Démoulin et al. (1997) in using a field model to analyze our particular region of interest between ARs 10486 and 10488/10493 (Fig. 2b).

For this, we adopted the Source Method with some modification. We modeled the observed field by a large number of magnetic monopoles arranged in a surface 10 arcsec below the photosphere. We then chose a certain limit for positive and negative polarities. Values outside these limits were accepted as significant for the field structure. Values in the interval were set to Zero. The effect was a defined reduction in the number of model monopoles, but some parts of the magnetogram were lost due to low field values which indeed turned out to be essential for representing the coronal field configuration. For this judgement, we compared the model field line pattern with the potential field extrapolation shown in P1. The low field subregions were retreated with reduced limits for the flux values. The pole strength and the polarity are obtained from the MDI data. The flux balance is achieved by a suitable choice of the limiting flux levels. We obtained a 106-polar and irregularly gridded magnetic monopole representation shown in Fig. 3a, plus signs and dark dots. With this model the field lines can subsequently be traced passing any footpoint grid in the photosphere. The $N(x, y)$-pattern is plotted grey-scaled in Fig. 3a. Dark shading means $N_{\max }$, white depicts $N(x, y)=0$. 


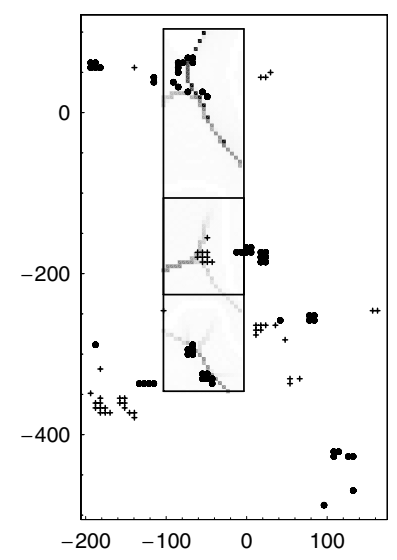

a)

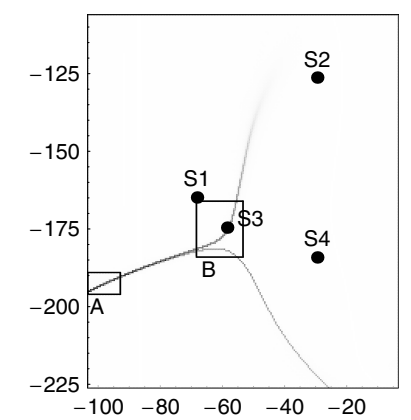

b)

Fig. 3. $N(x, y)$ after Eq. (1). Dark shading means $N_{\max }$, white is $N=0$. Axes in arcsec, $\mathrm{N}$ is top, $\mathrm{E}$ is left. a) $N(x, y)$ plotted in three regions (between ARs 10486 and 10488) according to the monopole model of the central part of the magnetogram Fig. 1a. The symbols depict monopoles: "+" means white, dark dots mean dark-plotted polarity in Fig. 1a. b) The small central box in panel a): Y-shaped displacement gradient pattern near the DMCS. Dots: DMCS centroids at NRH frequencies: S1\&S2-327 MHz; S3-410.5 MHz; S4-432 MHz. Boxes (A) and (B) are considered in Fig. 4.

A comparison of the displacement gradient calculated from the potential field extrapolation of the MDI magnetogram and the same measure for the model field lines confirms that the $N(x, y)$-graphs of the original and the model data resemble each other. This underlines that the selected monopole system is representative of the SOHO-MDI 11:11 UT observations.

We obtain large $N(x, y)$-values for locations near ARs 10486 and 10488. Special attention must be devoted to the middle part of the stripe in Fig. 3a. There is a Y-shaped $N(x, y)$-pattern east of AR 10491 wrapping around its trailing polarity. In Fig. 3b the Y-pattern of high connectivity gradient values is enlarged, boxes A and B define subregions of the pattern. The source centroids of the DMCS are overplotted as black dots S1‥S4. Although the position in Fig. 1b (white asterisk) is integrated over time and at $327 \mathrm{MHz}$, we provide it here more in detail. The $327 \mathrm{MHz}$ source is split (S1, S2), and the 410.5 (S3) and $432 \mathrm{MHz}$ (S4) source sites of the continuum are also included. In projection, the centroids $\mathrm{S} 1, \mathrm{~S} 3$ and $\mathrm{S} 4$, time-integrated positions measured at three independent observing frequencies, are arranged perpendicularly to a line of extreme $N$-values. S1 and S2, both at $327 \mathrm{MHz}$, are placed on different sides along that line.

In the boxes A and B of Fig. $3 b$ we use now a 0.5 arcsec field line footpoint spacing in the photosphere. The results are shown in Fig. 4. Colored dots are field line footpoints, their connectivity is color-coded. Shaded are the pixels with a high connectivity gradient, a pixel is darkest if its right or upper neighbor belong to another flux system.

We find that from the Y-pattern in the displacement data a bundle of field lines leads toward the North (red dots, to AR 10488), another one points toward the South (blue dots, to the flaring AR 10486). Both the red and the blue flux systems are bound by the Y-pattern. In the space spanned by the Y, a third flux system extends toward the leading part of AR 10491 (green dots). It is impressive that - in the model field - the green system penetrates far into the narrow stick of the Y-pattern (Fig. 4a) thus stretching out into a range of very low longitudinal magnetic field values. The model field structure of these three separatrices

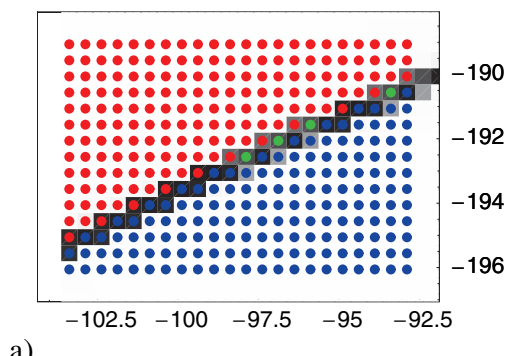

a)

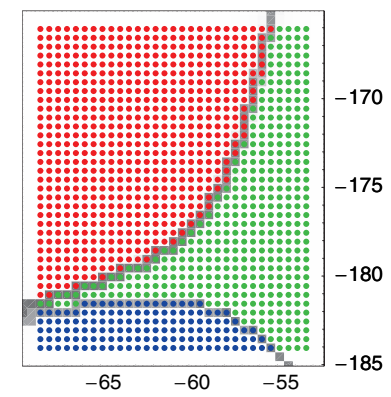

b)

Fig. 4. The Y-shaped pattern in $N(x, y)$ of Fig. 3b, boxes (A) and (B), with photospheric (model) field line footpoints of a $0.5 \operatorname{arcsec}$ grid. The flux cells are color-coded: toward AR 10488 - Red, AR 10486 - Blue, AR 10491 - Green. Grey-shaded are pixels with extreme displacement gradient (see text). a) The eastern part of the $\mathrm{Y}$ with the widest-east extended green flux-cell footpoints. b) The Y branch point.

converging in a range of low longitudinal field, is expected to be very sensitive to external disturbances. Mandrini et al. (2002) reported on arch filament system and surge formation at two interacting bald patch separatrices.

\section{Discussion and conclusions}

We found that the red-green, the blue-green, and the red-blue separatrices approach each other in the studied Y-pattern of the displacement data. Between the separatrix surfaces, highly concentrated currents can flow if the static potential field is slightly disturbed somewhere. Evidently such a situation must be expected during the X17 flare in AR 10486 and the related release of a halo CME (P1) even because one of the closely touching flux cells near the DMCS leads to the flaring AR 10486. The short flash of the DMCS during the onset of the impulsive phase (Fig. 2a, 11:01:30-11:02:45 UT) reveals the early activation of the field at this location, and the later strong emission (11:13-11:19 UT) confirms the persistence and stability of the situation despite of the disturbance.

Figure 5 gives a top and a perspective view on the system of those field lines which are rooted in the grey-shaded pixels of Fig. 4a. This means we see the most eastward extended green flux cell field lines as well as immediate red and blue neighbors. The $N(x, y)$-pattern of Fig. $3 \mathrm{~b}$ is underlaid grey-shaded. The radio sources are inserted from Fig. 3b (bold black dots) according to the independently determined five-fold Newkirk (1961) density model. The high frequency DMCS $(432,410.5 \mathrm{MHz})$ are situated below, the low frequency $(327 \mathrm{MHz})$ DMCS are situated above the shells formed by the green- and red-plotted potential field lines ${ }^{2}$, and are aligned perpendicularly (at three

\footnotetext{
2 Source heights for 432, 410 and $327 \mathrm{MHz}$ are 47.8, 56.1, and $94.9 \mathrm{Mm} ; \approx \pm 5 \mathrm{Mm}$.
} 

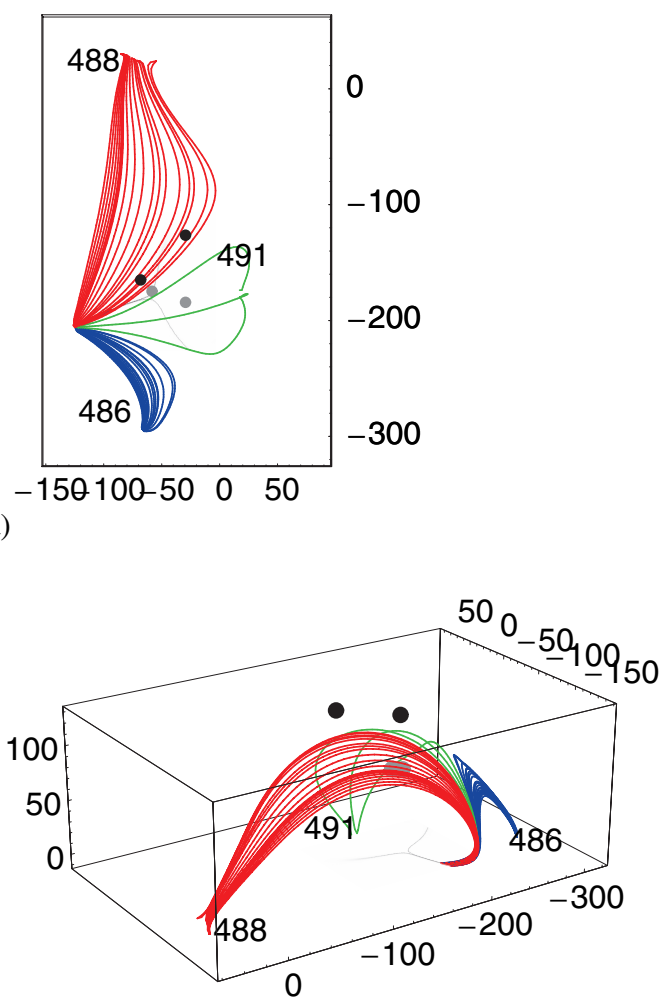

b)

Fig. 5. Field lines stemming from Fig. 4a, grey pixels only. Scales in arcsec. a) Top view. b) Perspective view from NE. Colors give the fluxcell association. Overplotted are the Y-pattern and the coronal radio sources as bold dots from Fig. 3b. AR numbers are given to connect this figure with Fig. 1a.

independent frequencies) and in parallel (at $327 \mathrm{MHz}$ ) with the green-red cell boundary.

The field lines of the different flux cell separatrices run in parallel near the DMCS centroids. If equally directed fieldparallel currents flow along the lines, then reconnection between lines of different flux cells is indeed possible (see e.g. the coalescence mode in Vršnak 2003). In this case, parts of different active regions are shortcircuited along interconnecting magnetic field lines. As a consequence of potential differences between the newly connected footpoints, a huge electric field strength can occur leading to particle acceleration acting for electrons and protons as well. This means it is probable that the nonthermal electrons feeding the DMCS radio emission are accelerated near the DMCS site. The fact that the source position is away from the flaring active region may be due to the sensitive converging separatrix pattern of the complex structured potential coronal magnetic field. It also comprises a magnetic connection to the flaring AR 10486. Of course we cannot completely exclude other explanations. An interesting aspect of our result is whether the presence of a remote separate source site can be the key to understanding the strange separation between electron- and protondriven sources discovered by the RHESSI gamma-ray imaging observations (Hurford et al. 2006).

Acknowledgements. H.A. thanks Dr. A. Hofmann for supplying the potential field extrapolation. We acknowledge the use of the Nançay Radio Heliograph, the SOHO Michelson Doppler Imager and Extreme Ultraviolet Telescope, and of the GOES satellites data. SOHO is a project of international cooperation between ESA and NASA. The work of G.R. is supported by Grant No. AU 106/13-2 of the Deutsche Forschungsgemeinschaft.

\section{References}

Aurass, H., \& Mann, G. 2004, ApJ, 615, 526

Aurass, H., Mann, G., Rausche, G., \& Warmuth, A. 2006, A\&A, 457, 681 (P1) Démoulin, P., Hénoux, J. C., Priest, E. R., \& Mandrini, C. H. 1996, A\&A, 308, 643

Démoulin, P., Bagala, L. G., Mandrini, C. H., Henoux, J. C., \& Rovira, M. G. 1997, A\&A, 325, 305

Gorbachev, V. S., \& Somov, B. V. 1988, Sol. Phys., 117, 77

Hurford, G. J., Krucker, S., Lin, R. P., et al. 2006, ApJ, 644, L93

Kerdraon, A., \& Delouis, J. 1997, in Coronal Physics from Radio and Space Observations, ed. G. Trottet (Heidelberg: Springer), 192

Klassen, A., Krucker, S., Kunow, K., et al. 2005, JGR, 110, A09S04

Mandrini, C. H., Démoulin, P., Schmieder, B., Deng, Y. Y., \& Rudawy, P. 2002, A\&A, 391, 317

Mann, G., Aurass, H., Voigt, W., \& Paschke, J. 1992, ESA SP-348, 129

Mann, G., Aurass, H., \& Warmuth, A. 2006, A\&A, 454, 969

Melrose, D. B. 1985, Solar Radiophysics, ed. D. J. McLean, \& N. R. Labrum (Cambridge: Cambridge University Press), 177

Newkirk, G. A. 1961, ApJ, 133, 983

Somov, B. V. 2000, Cosmic Plasma Physics, Astrophys. Space Sci. Lib. 251 (Kluwer: Dordrecht)

Somov, B. V., Kosugi, T., Hudson, H. S., Sakao, T., \& Masuda, S. 2003, Adv. Sp. Res., 32, 2439

Spicer, D. S. 1977, Sol. Phys., 53, 305

Titov, V. S., Hornig, G., \& Démoulin, P. 2002, JGR, 107, 10.1029/2001JA000278

Vršnak, B. 2003, in Energy Conversion and Particle Acceleration in the Solar Corona?, ed. K.-L. Klein, LNP 612 (Springer), 28 